\title{
Hamiltonian Dynamics of the Landau-Ginzburg Model for the Phase Transition in $\left(\mathrm{NH}_{4}\right)_{2} \mathrm{Be} \mathrm{F}_{4}$
}

\author{
A. Ribeiro Filho, R. C. de Miranda Filho, D. S. de Vasconcelos and J. F. M. Rocha \\ Instituto de Física, Universidade Federal da Bahia, \\ Campus Universitário de Ondina, \\ 40210-340, Salvador, BA, Brazil
}

Received 9 May, 2000

\begin{abstract}
We present a Hamiltonian treatment of the Landau-Ginzburg theory of phase transitions in the case of incommensurate ferroelectric systems like $\left(\mathrm{NH}_{4}\right)_{2} \mathrm{BeF}_{4}$. This formulation has the advantage of treating the system as an example of a nonlinear dynamical system.
\end{abstract}

\section{Introduction}

Analytical peculiarities of the nonlinear problems that arise in the incommensurate structures are not, in general, straightforward and require sometimes alternative methods, which can clarify the behaviour, of the main properties of different systems as improper ferroelectrics, chiral smectic ferroelectric liquid crystals, and other. The great interest has been to study the distinct phase transitions in incommensurate systems without the use of any ansatz a priori. The problem is not simple as have been shown by well established numerical works involving these structures. McMillan, Ishibashi, Dzyaloshinskii and other authors[1] have used the known constant-amplitude ansatz (of the order parameter) or PMO-ansatz (phase-modulated-only ansatz) or solitonic hypothesis, in order to study the free energy density of the system, and to write the optimized equations. Normally, it arises as a nonlinear set of coupled Euler-Lagrange equations (in terms of amplitude and phase of the order parameter), without any analytical solutions. McMillan's hypothesis, instigated the arising of the descommensurations array (or phase solitons) which allowed a best understanding of the incommensurate systems.

One of the first attempts to introduce an alternative treatment in order to study the mentioned problem was proposed by Ribeiro Filho et al [2], through a Lagrangian treatment involving analogies between the "time" evolution of the order parameter components, in the Landau-Ginzburg theory, of the chiral smectic ferroelectric liquid crystal $D O B A M B C$ $(1-n-$ decyloxybenzylidene $-p /$ - amino $-2-$ methylbutycinnamate) and the usual configuration space of a particle in a two-dimensional framework. Another example of such methods was introduced by Golovko [3] who presented a classical approach in or- der to study incommensurate structures in thiourea and other ferroelectrics systems. In recent years several works [4] involving a Hamiltonian treatment, in order to study the liquid crystal $D O B A M B C$, have roused the interest of experimentalists and theoreticians. In this work, it is presented a similar treatment for the improper ferroelectric system $\left(\mathrm{NH}_{4}\right)_{2} \mathrm{BeF}_{4}$. In section II we describe the mentioned ferroelectric and some aspects of the canonical formalism applied to it. In section III, we perform some analytical calculations involving the stability analysis and the fixed points linked to the problem. Finally, in the concluding section, it is discussed the main results of this work.

\section{Theory}

In this section we introduce an alternative formulation of the Landau-Ginzburg model of phase transitions[4] for the case of the improper ferroelectric $\left(\mathrm{NH}_{4}\right)_{2} \mathrm{BeF}_{4}$, which belongs to the chemical family $A_{2} B X_{4}[5]$. In order to construct the dynamical theory, which allow to study the "time" evolution of the order parameter components, in the mentioned Landau-Ginzburg theory, we consider, at first, the correlation between the configuration space $\left(q_{1} \ldots, q_{n}\right)$, with that one of the order parameter components $\left(\xi_{1}, \ldots, \xi_{n}\right)$. We can write the expression of the Hamiltonian density $\mathcal{H}$ in terms of the free energy density $f$ as

$$
\mathcal{H}=\sum_{j} \dot{\xi}_{j} p_{j}-f
$$

where $p_{j}$ is the canonical momentum conjugated to $\xi_{j}$.

From the canonical formalism we can write the Hamilton's differential equations: 


$$
\dot{\xi}_{j}=\frac{\partial H}{\partial p_{j}} ; \dot{p}_{j}=-\frac{\partial H}{\partial \xi_{j}},(j=1, \ldots, n)
$$

We study here the solutions of these canonical equations rewriting them in a single equation, that is, we combine the vectors $p$ and $\xi$ into a $2 n$-dimensional vector $x=(p, \xi)$. It is possible to interpret the quantities $\left(\frac{\partial H}{\partial p_{j}}, \frac{\partial H}{\partial \xi_{i}}\right)$ as a $2 n$-dimensional vector $\nabla H$, and will introduce a $2 n \times 2 n$ matrix $\mathbf{J}$ :

$$
\mathbf{J}=\left(\begin{array}{cc}
0 & -\mathbf{I} \\
\mathbf{I} & 0
\end{array}\right)
$$

being $\mathbf{I}$ the $n \times n$ identity matrix. From such premises it is possible to rewrite the Hamilton's equations unified in the form $\mathbf{J} \cdot \dot{x}=-\nabla H(x)$, where $H$ is the Hamiltonian function and the state of the system is determined by the vector $x=\left(x_{1}, \ldots, x_{2 n}\right)$, so that the set of these vectors constitutes the phase space of the system, $\mathcal{M}=\{x\}$, which, in the general case, corresponds to a given manifold. In particular case as $\mathcal{M}=\mathbf{R}^{2 n}$, we define the scalar product $(x, y)=\sum_{i=1}^{2 n} x_{i} y_{i}$. Using the matrix $\mathbf{J}$ it is possible to define a Poisson bracket structure on the space $\mathcal{F}(\mathcal{M})$ of smooth functions $\left(C^{\infty}\right)$ on $\mathcal{M}$, that is,

$$
\{Q(x), P(x)\}=-(\nabla Q, J \nabla P)=\sum_{i=1}^{n}\left(\frac{\partial Q}{\partial p_{i}} \frac{\partial P}{\partial \xi_{i}}-\frac{\partial Q}{\partial \xi_{i}} \frac{\partial P}{\partial p_{i}}\right)
$$

which satisfies the Jacobi identity: $\{Q,\{P, R\}\}+$ $\{P,\{R, Q\}\}+\{R,\{Q, P\}\}=0$, as well as the relation $\{Q(x), P(x)\}=-\{P(x), Q(x)\}$, and the Leibniz rule: $\{Q, P R\}=\{Q, P\} R+\{Q, R\} P$. The two first relations also define a Lie algebra structure on $\mathcal{F}(\mathcal{M})$. So, the mentioned Hamilton's canonical equations can be written as,

$$
\dot{x}=\{H, x\}=X
$$

which is the canonical form of the mentioned equations of motion of a Hamiltonian system, which is characterized by $\{\mathcal{M},\{\},, H(x)\}$, that is, by the phase space $\mathcal{M}$, the Poisson structure $\{$,$\} and the Hamiltonian func-$ tion $H(x) . X$ is the so-called Hamiltonian vector field associated with the Hamiltonian function $H(x)[6]$.

Ferroelectric materials besides exhibit a spontaneous electric dipole momentum, can also be defined as substances in which is characterized a relation between the polarization and the electric field through a hysteresis cycle. One of the most important crystal families that presents the ferroelectricity phenomenom is characterized by the chemical formula $A_{2} B X_{4}$, of the so-called improper ferroelectics [5], which can present other order parameters besides the polarization. The ferroelectric $\left(\mathrm{NH}_{4}\right)_{2} \mathrm{BeF}_{4}$ (Ammonium fluorberyllatte) is an important member of that molecular family and presents, like other incommensurate ferroelectrics, a sequence of two phase transitions involving three distinct phases: the paraelectric (or normal or prototype) or high temperature phase $(\mathrm{N})$, the incommensurate phase (I), where the transition temperature is $T_{i}=-90.0^{\circ} \mathrm{C}$, and, finally, the ferroelectric commensurate phase (C) which begins at temperature $T_{c}=-96.0^{\circ} \mathrm{C}$. The symmetry of the mentioned crystal in the $(\mathrm{N})$ phase is or- thorrombic $D_{2 h}^{16}-P c m m$, the (C) phase is also orthorrombic $C_{2 \nu}^{9}-P n 2_{1} a$, and the intermediary (I) phase is indetermined, with an incommensurate modulation in the a-axis of the structure.[1]'[5] The polar axis in the $(\mathrm{C})$ phase is parallel to the b-axis, and the lattice parameter of the a-axis is doubled[7]. Experiments using magnetic resonance have contributed for the best understanding of the main physical features of the different phases of this material when are considered the high and low temperature regions in the (I) phase. It has been established that in the region near the normal phase, the wave of incommensurate modulation can be described as a "plane wave", and as the temperature leads to $T_{c}$, of the lock-in (incommensurate commensurate) transition, the approximated commensurate regions are present, but separated by static solitonic lattices so-called discommensurations or domain walls $[1],[5]$ where the phase of the modulation wave vary faster that one in the case of a plane wave. In reference to the (I) phase it is worth to emphasize that the soft mode condensation at temperature $T_{i}$, in the mentioned crystal, leads to the modulation of the parent lattice for an incommensurate structure whose periodicity is an irrational multiple of the period of the prototype lattice. The characterization of this (I) phase can not be obtained by one of the 230 space groups but it can be studied by the superspace group formalism.[5]

Considering the Landau-Ginzburg theory of phase transitions of the ferroelectric $\left(\mathrm{NH}_{4}\right)_{2} \mathrm{BeF}_{4}$, it is possible to establish the free energy density potential for this crystal and isomorphous [8] as

$$
f(x)=f_{h}+f_{n h}+f_{L}
$$

where the indexes $h, n h$ and $L$, means the homoge- 
neous, inhomogeneous parts and the Lifshitz invariant of the mentioned thermodynamical potential, that is,

$$
\begin{gathered}
f_{h}=\frac{1}{2} \alpha\left(\xi_{1}^{2}+\xi_{2}^{2}\right)+\frac{1}{4} \beta\left(\xi_{1}^{2}+\xi_{2}^{2}\right)^{2}+\frac{1}{2}(\gamma-\beta) \xi_{1}^{2} \xi_{2}^{2} \\
f_{n h}=\frac{1}{2} \kappa\left[\left(\frac{d \xi_{1}}{d x}\right)^{2}+\left(\frac{d \xi_{2}}{d x}\right)^{2}\right]
\end{gathered}
$$

and

$$
f_{L}=\delta\left(\xi_{1} \frac{d \xi_{2}}{d x}-\xi_{2} \frac{d \xi_{1}}{d x}\right)
$$

where $x$ is the coordinate and $\xi_{j}(j=1,2)$ are the order parameter components which transform in according to a two-dimensional irreducible representation in $X\left[k_{o}=\left(\frac{\pi}{a}, 0,0\right)\right]$ point on the Brillouin zone boundary, and the coefficient $\alpha=\alpha_{o}\left(T-T_{o}\right)$ depends linearly on temperature and it is the so-called Landau parameter. The other parameters are: $\beta>|\gamma|, \kappa>0$ and $\delta$ are considered temperature independent. The Lifshitz term (8) is allowed by symmetry and its existence prohibits the second-order transition directly into the commensurate phase $(\mathrm{C})$ from the normal phase $(\mathrm{N})$. Another important feature of this material is that when temperature decreases, the coefficient of the quadratic terms in $\xi_{j}(j=1,2)$ becomes zero for the critical wavenumber $k_{i}=\frac{|\delta|}{\kappa}$, at temperature $T_{i}$, given by $\alpha_{i}=\alpha_{o}\left(T_{i}-T_{o}\right)=\frac{\delta^{2}}{\kappa}$, where a second-order normalincommensurate transition takes place[8]. The aim of this work is to discuss some qualitatively fundamental aspects of the physics of the mentioned ferroelectric, from a Hamiltonian approach based upon a simple one-dimensional Landau-Ginzburg model given by (5). In this equation the expression $(\beta-\gamma) \beta^{-1}$ measures the anisotropy factor of the thermodynamical potential $f$. It is worth to stress that just below the temperature $T_{i}$, the anisotropic part of (5) can be neglected in reference to the isotropic one and therefore the solution of the problem is given by the sinusoidal functions $\xi_{j}(j=1,2)$, as we will show in the next section. Fig. (1) shows the homogeneous part of the thermodynamical potential in terms of the order parameter $\xi_{o}$, when it is considered the isotropic case $(\beta=\gamma)$. Using some numerical values quoted in literature [8], it is sketched in Figs. (2) and (3), for $\beta=0.8$ and $\gamma=0.2$, the homogeneous part of the thermodynamical potential. In this case the anisotropy is not quite large, so that the incommensurate phase is stable down to more large range of temperature. Figs. (4) and (5), where we use $\beta=0.8$ and $\gamma=-0.4$, indicate the case when the anisotropy is large enough and, in consequence, the contour lines deviate from the circular pattern more clearly than in Fig. (3). In this case the temperature range of the incommensurate phase is quite narrow because, as well established, the anisotropy energy, that favours the commensurate phase, becomes prominent part of the total energy on close and below $T_{i}$.

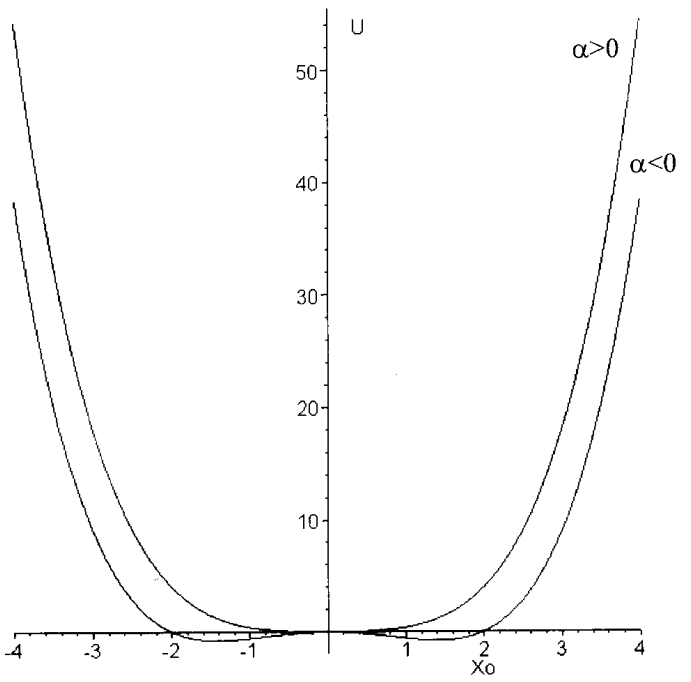

Figure 1. Thermodynamical potential $U \equiv f_{h}$ versus order parameter $\left(x_{o} \equiv \xi_{o}\right)$, for $\alpha=0.8$ and $\alpha=-1.6 ; \beta=0.8$.

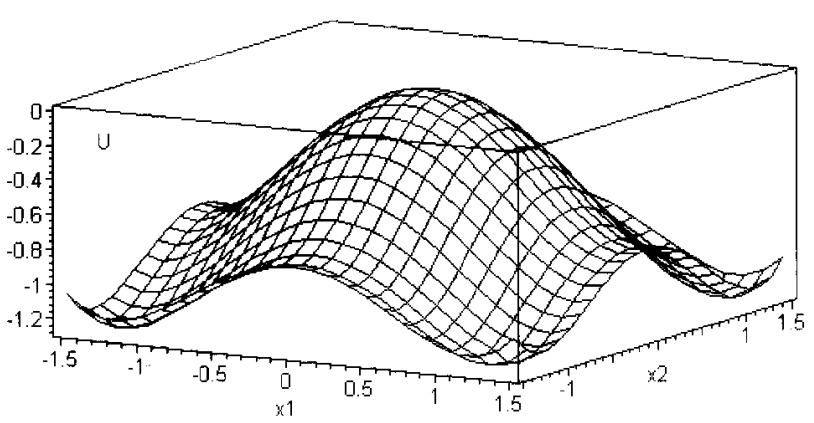

Figure 2. Thermodynamical potential $U\left(x_{1}, x_{2}\right), x_{1} \equiv \xi_{1}$ e $x_{2} \equiv \xi_{2}$, for $\alpha=-1.6, \beta=0.8$ and $\gamma=0.2$.

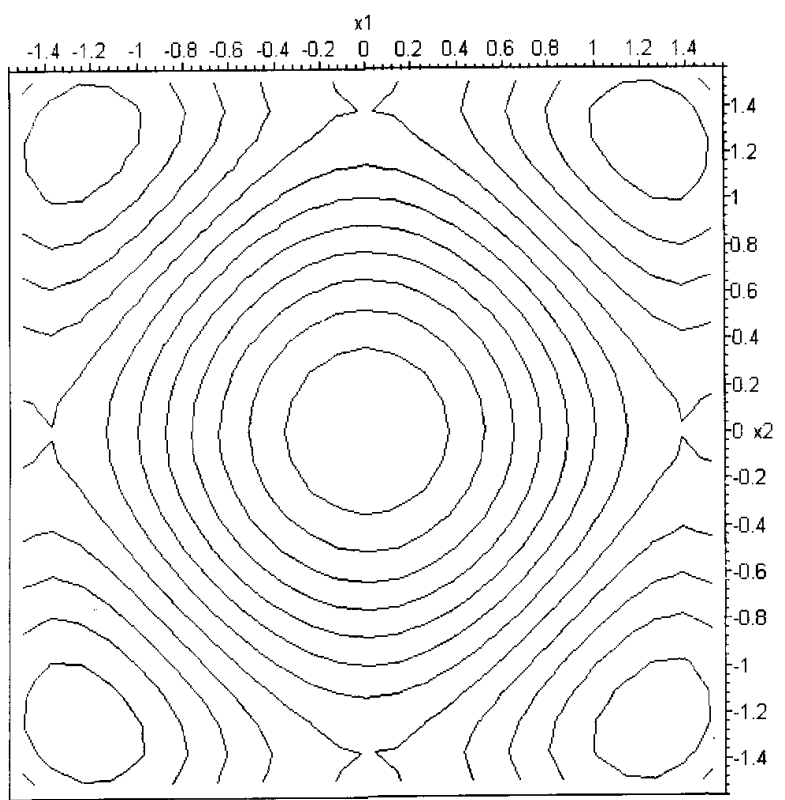

Figure 3. Contour lines of the homogeneous potential for $\alpha=-1.6, \beta=0.8$ and $\gamma=0.2$. 


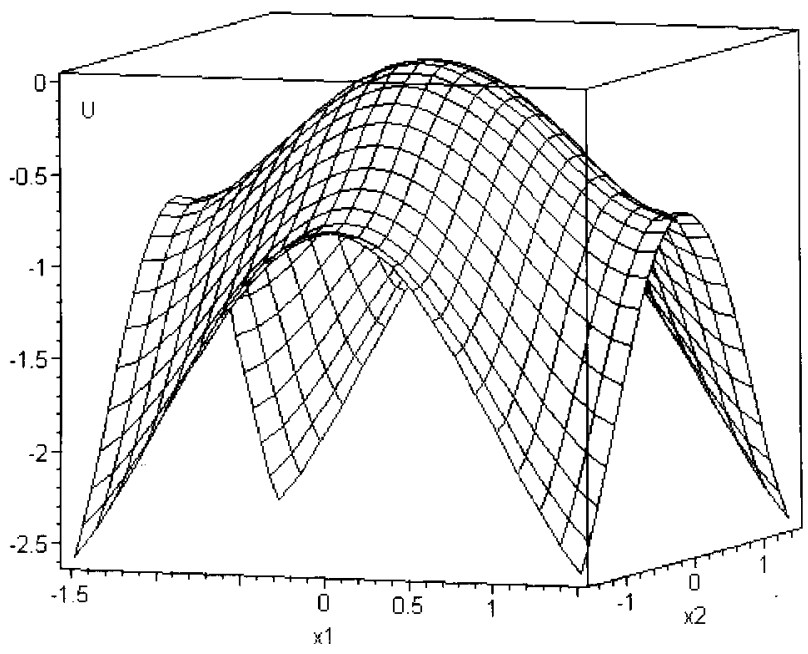

Figure 4. Thermodynamical potential $U\left(x_{1}, x_{2}\right), x_{1} \equiv \xi_{1}$ e $x_{2} \equiv \xi_{2}$, for $\alpha=-1.6, \beta=0.8$ and $\gamma=-0.4$.

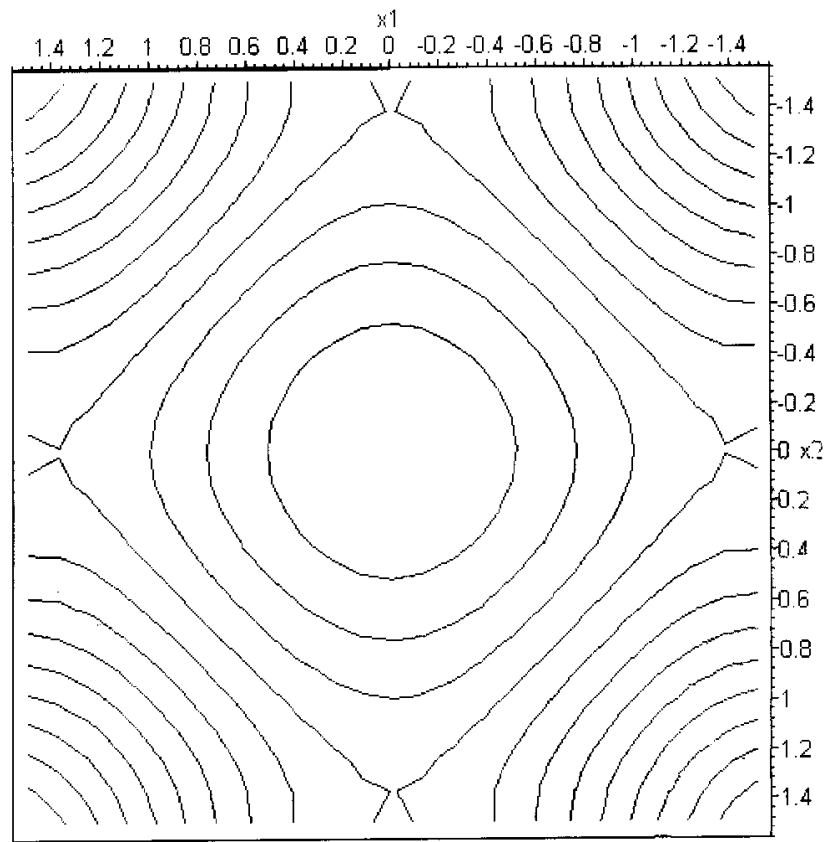

Figure 5. Contour lines of the homogeneous potential for $\alpha=-1.6, \beta=0.8$ and $\gamma=-0.4$.

In order to make easy the computations involving (1) it will be considered the following analogies in (5): $x$ like "time" $t ; f \rightarrow \mathcal{L}$ (the Lagrangian), where $\mathcal{L}=T-V$, being $T$ and $V$ the kinetic and potential energies, respectively. In this mechanical analogy $T \rightarrow f_{n h}$ and $V=U+U_{L}$, with $U \rightarrow f_{h}$ and $U_{L} \rightarrow f_{L}$ in the generalized potential depending on the "velocities " $\dot{\xi}_{j}(j=1,2)$. Using such designations we are able calculate the canonical momenta by

$$
p_{j}=\kappa \dot{\xi}_{j}+(-1)^{j} \delta \xi_{j+1}
$$

with $(j=1,2 ; 3 \equiv 1)$ and through a usual Legendre transformation we can write the following Hamiltonian

$$
\begin{gathered}
H=\frac{1}{2 \kappa}\left(p_{1}^{2}+p_{2}^{2}\right)+\frac{1}{2} \Delta\left(\xi_{1}^{2}+\xi_{2}^{2}\right)-\frac{1}{4} \beta\left(\xi_{1}^{2}+\xi_{2}^{2}\right)^{2} \\
-\frac{1}{2}(\gamma-\beta) \xi_{1}^{2} \xi_{2}^{2}+\Lambda\left(p_{1} \xi_{2}-p_{2} \xi_{1}\right)
\end{gathered}
$$

where $\Delta=\frac{\delta^{2}}{\kappa}-\alpha$ and $\Lambda=\frac{\delta}{\kappa}$. When $\gamma \neq \beta$, the anisotropic term makes the phase and the amplitude, of the order parameter, be $x$-dependent, that is $\xi_{j}(j=1,2)$ begin to include higher harmonics where a given critical wavenumber $k_{i}$ is determined in order to minimize

$$
F=\frac{1}{L} \int f(x) d x
$$

where $L$ is the "length" of crystal. It is worth to point out that the equation (10), with $\gamma \neq \beta$, also defines a nonlinear Hamiltonian system, which is nonintegrable[6]. We can rewrite the Hamiltonian (10) as

$$
H=H_{o}-\frac{1}{2}(\gamma-\beta) \xi_{1}^{2} \xi_{2}^{2}
$$

so that, if $\gamma=\beta$, the system with $H=H_{o}$ is integrable and isotropic, with

$$
G=\Lambda\left(p_{1} \xi_{2}-p_{2} \xi_{1}\right)
$$

being the second constant of motion besides the energy. This may be verified by direct calculation of the Poisson bracket $\{H, G\}=0$.

\section{Analytical Calculations}

From the equation (11), and using the Hamiltonian treatment, it is possible to get all results that characterize the different phase transitions in the mentioned ferroelectric crystal. The transition between $(\mathrm{N})$ and (I) phases can also be described by Hamiltonian (10), which gives rise, as we have showed in the section II, the following system of ordinary differential equations, which can be rewrite in short, $\mathbf{X}=\mathbf{F}(\mathbf{X})$, with $\mathbf{X}$ being the Hamiltonian vector field, and

$$
\begin{gathered}
\dot{x}_{1}=F_{1}\left(x_{1}, x_{2}, x_{3}, x_{4}\right)=\kappa^{-1} x_{3}+\Lambda x_{2} \\
\dot{x}_{2}=F_{2}\left(x_{1}, x_{2}, x_{3}, x_{4}\right)=\kappa^{-1} x_{4}-\Lambda x_{1} \\
\dot{x}_{3}=F_{3}\left(x_{1}, x_{2}, x_{3}, x_{4}\right)=-\Delta x_{1}+\Lambda x_{4}+\gamma x_{1} x_{2}^{2}+\beta x_{1}^{3}
\end{gathered}
$$

$$
\dot{x}_{4}=F_{4}\left(x_{1}, x_{2}, x_{3}, x_{4}\right)=-\Delta x_{2}-\Lambda x_{3}+\gamma x_{1}^{2} x_{2}+\beta x_{2}^{3}
$$

where we substitute $\xi_{1}$ by $x_{1} ; \xi_{2}$ by $x_{2} ; p_{1}$ by $x_{3}$ and $p_{2}$ by $x_{4}$. The set of equations showed above is an example 
of a dynamical system, where the fixed points can be determined by condition $\mathbf{F}(\mathbf{X})=0$. The first of these points $P_{1}:(0,0,0,0)$ is quite interesting in our present discussion as we will see hereafter. After performing the stability analysis around this point it is possible to recognize several experimental features of the mentioned ferroelectric, as we have pointed out in the last section.

Following the standard procedure [6] of such analysis in a dynamical system, that is, using a linear approximation around the equilibrium point $P_{1}$ we get

$$
\dot{\mathbf{X}} \approx J_{H}\left(P_{1}\right) \mathbf{X}
$$

where the Jacobian matrix, $J_{H}\left(P_{1}\right)$, is given by

$$
J_{H}\left(P_{1}\right)=\left(\begin{array}{cccc}
0 & \Lambda & \kappa^{-1} & 0 \\
-\Lambda & 0 & 0 & \kappa^{-1} \\
-\Delta & 0 & 0 & \Lambda \\
0 & -\Delta & -\Lambda & 0
\end{array}\right)
$$

The four eigenvalues of the characteristic equation are obtained, in this approximation, by

$$
\begin{gathered}
\operatorname{det}\left(J_{H}\left(P_{1}\right)-\lambda \mathbf{I}\right)=\lambda^{4}+2 \lambda^{2}\left(\Lambda^{2}+\kappa^{-1} \Delta\right) \\
+\left(\Lambda^{4}-2 \kappa^{-1} \Delta \Lambda^{2}+\kappa^{-2} \Delta^{2}\right)=0
\end{gathered}
$$

and the solutions are

$$
\begin{aligned}
& \lambda_{1}=-\lambda_{2}=\left[-\kappa^{-1}\left(\Delta+\delta^{2} \kappa^{-1}\right)+2 \delta \kappa^{-\frac{3}{2}} \Delta^{\frac{1}{2}}\right]^{\frac{1}{2}} \\
& \lambda_{3}=-\lambda_{4}=\left[-\kappa^{-1}\left(\Delta+\delta^{2} \kappa^{-1}\right)-2 \delta \kappa^{-\frac{3}{2}} \Delta^{\frac{1}{2}}\right]^{\frac{1}{2}}
\end{aligned}
$$

where $\Delta=0$ corresponds to the structural stability breaking and we get the critical temperature, $T_{i}=$ $T_{o}+\frac{\delta^{2}}{\alpha_{o} \kappa}$. This temperature is therefore the normalincommensurate transition temperature[8] and for this case, after some algebraic calculations, the eigenvalues are pure imaginary, that is, $\lambda= \pm i \frac{|\delta|}{\kappa}$ and the corresponding eigenvectors are

$$
x_{o j}=\mathcal{A}\left(\begin{array}{c} 
\pm i \\
1 \\
0 \\
0
\end{array}\right)
$$

where $x_{o j}(o j=1,2)$ corresponds to $\pm i$ in (23). Finally, we get: $x_{1}=\mathcal{A} \cos \frac{|\delta|}{\kappa} x$ and $x_{2}=\mathcal{A} \operatorname{sen} \frac{|\delta|}{\kappa} x$, that is, such expressions show that the order parameter components $\xi_{j}(j=1,2)$, of this ferroelectric system, are modulated with the critical wavelength $k_{i}=$ $\frac{|\delta|}{\kappa}$. These results characterize the onset of the incommensurate structure in this material and they are in agreement with those ones obtained, traditionally, by other authors[8], and show that there is a continuous transition at $T_{i}$ between the $(\mathrm{N})$ and $(\mathrm{I})$ phases. The wavelength of the modulation is not a simple multiple of the lattice period in the normal phase.

From the set of equations (14)-(17) it is simple to find the following nine fixed points, with coordinates $P_{i}\left(x_{1}^{*}, x_{2}^{*}, x_{3}^{*}, x_{4}^{*}\right)$, of the dynamical system: $P_{1}(0,0,0,0) ; \quad P_{2}(0, n,-\delta n, 0) ; P_{3}(0,-n, \delta n, 0)$; $P_{4}(n, 0,0, \delta n) ; P_{5}(-n, 0,0,-\delta n) ; P_{6}(m, m,-\delta m, \delta m)$; $P_{7}(m,-m, \delta m, \delta m) ; \quad P_{8}(-m, m,-\delta m,-\delta m)$ and the last point $P_{9}(-m,-m, \delta m,-\delta m)$; where $n=\left(\frac{-\alpha}{\beta}\right)^{\frac{1}{2}}$ and $m=\left(\frac{-\alpha}{\beta+\gamma}\right)^{\frac{1}{2}}$. The analysis of the last eight fixed points $\left(P_{2}, \ldots, P_{9}\right)$ is quite similar to that one employed in $P_{1}(0,0,0,0)$ in spite of the algebraic work be quite extended. Normally, we consider again the linearized system $J_{i j}=\frac{\partial F_{i}}{\partial x_{j}}=F_{i}^{j}$, so that $\mathbf{J}=\left[J_{i j}\right]$ and using the equations (14)-(17) we find: $F_{1}^{1}=0$; $F_{1}^{2}=\Lambda ; F_{1}^{3}=\kappa^{-1} ; F_{1}^{4}=0 ; F_{2}^{1}=-\Lambda ; F_{2}^{2}=0 ;$ $F_{2}^{3}=0 ; F_{2}^{4}=\kappa^{-1} ; F_{3}^{1}=-\Delta+\gamma x_{2}^{2}+3 \beta x_{1}^{2} \equiv A_{1}$; $F_{3}^{2}=2 \gamma x_{1} x_{2} ; F_{3}^{3}=0 ; F_{3}^{4}=\Lambda ; F_{4}^{1}=2 \gamma x_{1} x_{2} \equiv B ;$ $F_{4}^{2}=-\Delta+\gamma x_{1}^{2}+3 \beta x_{2}^{2} \equiv A_{2} ; F_{4}^{3}=0 ; F_{4}^{4}=-\Lambda$. Therefore the characteristic equation is given by

$$
\begin{gathered}
\lambda^{4}+\left[2 \Lambda^{2}-\left(A_{1}+A_{2}\right) \kappa^{-1}\right] \lambda^{2}+\Lambda^{4}+\kappa^{-2} A_{1} A_{2} \\
+\kappa^{-1} \Lambda^{2}\left(A_{1}+A_{2}\right)-\kappa^{-2} B^{2}=0
\end{gathered}
$$

where

$$
\begin{gathered}
A_{1}=-\Delta+\gamma x_{2}^{* 2}+3 \beta x_{1}^{* 2} \\
A_{2}=-\Delta+\gamma x_{1}^{* 2}+3 \beta x_{2}^{* 2} \\
B=2 \gamma x_{1}^{*} x_{2}^{*}
\end{gathered}
$$

and the main algebraic results of these calculations have been summarized and discussed in the appendix.

\section{Conclusions}

In this work we explicit the possibility in using the mathematical background of dynamical systems in order to discuss the phase transitions problem in the ferroelectric crystal $\left(\mathrm{NH}_{4}\right)_{2} \mathrm{BeF}_{4}$. This treatment can be extended to other members of the chemical family $A_{2} B X_{4}$. For this, it was introduced a Hamiltonian treatment, in addition to the mechanical analogy, considering the mentioned ferroelectric material as a typical Hamiltonian system.

Summarizing the discussion underlined in section III and appendix we have found four distinct values for the Landau parameter $\alpha: \alpha_{o}$ " $, 0, \alpha_{o}^{+}$and $\alpha_{o}^{-}$. In the neghbourhood (the left and right sides) of each different $\alpha$-value, the phase space presents different features. In the case $\alpha=\alpha_{o}$ ", it is observed, to the right side, that there is only a fixed point which is unstable hyperbolic. To the left side, of this $\alpha$-value, there is also 
a unique fixed point, which is elliptic. When $\alpha=0$, in both left and right sides, the fixed points are eliptic. For $\alpha>0$ there is only one critical point while for $\alpha<0$ (which is the more important case for the Landau-Ginzburg model) there are nine fixed points. In the case of $\alpha=\alpha_{o}^{+}$implicates that in the points $P_{1}, \ldots, P_{5}$ there is not change of behaviour. With reference to the points $P_{6}, \ldots, P_{9}$, the change is identical to those one in $\alpha_{o}$ " despite all hyperbolic points are localized in the left side while the elliptic ones in the right size. For $\alpha_{o}^{-}$we note that there are not changes for the points $P_{1}, \ldots, P_{5}$, while $P_{6}, \ldots, P_{9}$ are hyperbolic in both sides (left and right), but in the case $\alpha<\alpha_{o}^{-}$the eigenvalues are real, while for $\alpha>\alpha_{o}^{-}$they are complex. So, from the analysis above only two $\alpha$-values ( $\alpha_{o}$ " e $\alpha_{o}^{+}$) separate hyperbolic and elliptic points. It is worth to emphasize that the temperature associated with these two $\alpha$-values are those obtained by other authors [8] and corresponds to the presence of the phase transition in the mentioned ferroelectric material.

From the characteristic equation (20) and matrix (23) it is showed the arising of an incommensurate plane wave involving the order parameter components $\xi_{1}$ (or $\left.x_{1}\right)$ and $\xi_{2}\left(\right.$ or $\left.x_{2}\right)$ in terms of a critical wavenumber $k_{i}$, while the critical temperature between the $(\mathrm{N})$ and (I) phases has been obtained, quickly, when we have considered the stability analysis, in the linear approximation, around the point $P_{1}(0,0,0,0)$. Using the equations (34), (35), (28) and (29), from the appendix, we get

$$
\alpha_{o}^{+}=-\left(\frac{\delta^{2}}{\kappa}\right)(\gamma+\beta)\left[\beta+\left(\beta^{2}-\gamma^{2}\right)^{\frac{1}{2}}\right]^{-1}
$$

which corresponds to the critical temperature

$$
T_{o}^{+}=T_{o}-\left(\frac{\delta^{2}}{\alpha_{o} \kappa}\right)(\gamma+\beta)\left[\beta+\left(\beta^{2}-\gamma^{2}\right)^{\frac{1}{2}}\right]^{-1}
$$

where the commensurate phase becomes unstable with respect to spatially modulated solution. The wavelength of the modulation wave, it is computed from the modulus of the eigenvalues, in (31), that is,

$$
\lambda=\frac{|\delta|}{\kappa}\left[2\left(\beta^{2}-\gamma^{2}\right)^{\frac{1}{2}}\right]^{\frac{1}{2}}\left[\beta+\left(\beta^{2}-\gamma^{2}\right)^{\frac{1}{2}}\right]^{-\frac{1}{2}}
$$

It is interesting to point out that in spite of the critical temperature $T_{o}^{+}$has been previously computed[8], meanwhile that expression does not represent in fact the actual lock-in temperature $T_{c}$ which takes place, in reality, at much lower temperature than $T_{o}^{+}$. With reference to $\left(\mathrm{NH}_{4}\right)_{2} \mathrm{BeF}_{4}$ it is worth to stress that the lock-in phase transition temperature, can be obtained, with more accuracy, from (11), when the free energy density potential model includes other physical contributions like external fields [9]. Using such thermodynamical potential models it is found a first-order lock-in phase transition in this material.
In order to discuss our Hamiltonian treatment we decide to use an established and simple model because, normally, the lock-in temperature calculation is quite cumbersome, when it is considered the influence of an external electric field on incommensuratecommensurate (lock-in) phase transition in this ferroelectric material, which gives a good agreement with experiment.

In summary, we have got new analytical results as well as those ones obtained by other authors for $\left(\mathrm{NH}_{4}\right)_{2} \mathrm{BeF}_{4}$, using a simple and elegant Hamiltonian approach, showing the efficacy of this alternative formulation for studying phase transitions and other features of improper ferroelectrics. The great advantages of this analytical approach has been the straightforward way in getting informations as well as many physical properties can be discussed more clearly. In comparison with the standard formalism used by different writers [8] we can stress that this Hamiltonian treatment is more simple and avoid some hypothesis used by them.

Despite the simplicity of the thermodynamical potential model, used in this work, it shows, more clearly, the possibility in using the Hamiltonian treatment for the Landau-Ginzburg theory of phase transitions. This approach creates new possibilities in studying phase transitions employing nonlinear dynamical techniques which have been discussed in other branches of Physics. Another aspect already underlined before [4] is that using the mathematical recourses of the nonlinear dynamics it is possible to study, more carefully, the chaotic structures that are present, experimentally, in the neighbourhood of the lock-in phase transition in this material. From such evidences it will be possible to analyze the chaoticity, in terms of Poincarè maps, the Liapunov exponent, power spectrum and the correlation function.

In this work we have concentrated in studying some aspects of the critical points present in $\left(\mathrm{NH}_{4}\right)_{2} \mathrm{BeF}_{4}$ and in discussing the behaviour of the Landau parameter, which is dependent of temperature.

Acknowledgements - One of us (A. R. F.) would like to thank the support of the Brazilian agencies $\mathrm{CNPq}$ and CAPES.

\section{References}

[1] W. L. McMillan, Phys. Rev. 14, 1496 (1976); I. E. Dzyaloshinskii, Sov. Phys. JETP, 20, 3, 665-671 (1965); E. M. do Nascimento, D. S. de Vasconcelos, J. F. M. Rocha e A. Ribeiro Filho, Rev. Bras. Ens. Física, 20, 1, 11-18 (1998); Y. Ishibashi and H. Shiba, J. Phys. Jpn, 44, 801 (1978).

[2] A. Ribeiro Filho, D. R. Tilley, and B. Zeks, Phys. Lett. A 100, 247 (1984).

[3] V. A. Golovko, Sov. Phys. Solid State, 23 (6), 958-961 (1981); Sov.Phys.JETP, 60 (3), 624 (1984). 
[4] K. C. Mundim, P. Pitanga, and A. Ribeiro Filho, Notas de Física, CBPF-NF-045, 1-10 (1992); phys. stat. sol. (b) 179, 53 (1993); P. Pitanga, A. Ribeiro Filho, and K. C. Mundim, phys. stat. sol. (b) 183, 103 (1994); P. Pitanga, K. C. Mundim, and A.Ribeiro Filho, phys. stat. sol. (b) 197, 517 (1996).

[5] R. Lifshitz, Rev. Mod. Phys. 69, 4, 1181-1218 (1997); V. C. Costa, D. S. de Vasconcelos, E. M. do Nascimento e A. Ribeiro Filho, Rev. Bras. Ens. Física, 20, 3, 220-229 (1998).

[6] J. E. Mardsen, Lectures on Mechanics, London Math. Soc. Lect. Notes Ser. 174, Cambridge Univ. Press (1992); A. M. O. de Almeida, "Sistemas HamiltonianosCaos e Quantização", Ed. Unicamp, (1995); A. M. Peremolov, "Integrable Systems of Classical Mechanics and Lie Algebras", vol. 1, Ed. Birkhäuser (1990); N. FFerrara e C. P. C. do Prado, "Caos uma Introdução", Ed. Edgard Blücher Ltda. (1994); R. C. Miranda Filho, and R. F. S. Andrade, Phys. Lett. A 153, 2, 3, 101 (1991).

[7] J. Kobayashi, Y. Uesu, J. Ogawa, and Y. Nishiara, Phys. Rev. B, 31, 7, 4569-4575 (1985).

[8] A. P. Levanyuk, and D. G. Sannikov, Sov. Phys. Sol. State 18, 245 (1976); Y. Ishibashi, Ferroelectrics 24, 119-126 (1980); H. Shiba and Y. Ishibashi, J. Phys. Soc. Jap. 44, 5, 1592-1599 (1978); Y. Ishibashi and V. Dvorak, J. Phys. Soc. Jap. 44, 32 (1978).

[9] P. Prelovsek, A. Levstik and C. Filipic, Phys. Rev. B, 28, 9, 6610-6612 (1983); O. Hudak, J. Phys. C: Solid State Phys., 16, 2641-2658 (1983).

\section{Appendix}

The solution of (24) for the fixed points $P_{6}, \ldots, P_{9}$, explicited in the text through its coordinates $x_{1}^{*}, x_{2}^{*}$, $x_{3}^{*}, x_{4}^{*}$, shows that $x_{1}^{* 2}=x_{2}^{* 2}=m^{2}=\frac{-\alpha}{\beta+\gamma}$.So, from $(24)$ we find:

$$
\begin{gathered}
A_{1}=A_{2}=A=\left(\frac{-2}{1+\beta^{-1} \gamma}\right) \alpha-\frac{\delta^{2}}{\kappa} \\
B^{2}=\left[\left(\frac{\alpha}{\beta+\gamma}\right) 2 \gamma\right]^{2}=c^{2} \alpha^{2}
\end{gathered}
$$

Substituting (28) and (29) in (24) we have

$$
\lambda^{2}=\mathcal{Z} \pm D^{\frac{1}{2}}
$$

where

$$
\begin{gathered}
D=\frac{c^{2} \alpha^{2}}{\kappa^{2}}+4 b \alpha \frac{\Lambda^{2}}{\kappa}+4 \Lambda^{4} \\
\mathcal{Z}=\frac{-b \alpha}{\kappa}-2 \Lambda^{2} \\
b=\frac{2}{1+\gamma \beta^{-1}}
\end{gathered}
$$

In the case $D<0$ we see that $\lambda^{2}$ and $\lambda$ are complex, taking into account that the coefficient of $\alpha^{2}$ in (31) is positive, implicates that $D$ is negative for $\alpha$ localized between the roots of equation $D=0$, that is,

$$
\begin{aligned}
& \alpha_{D o+}=2 \kappa \Lambda^{2} c^{-2}\left[-b+\left(b^{2}-c^{2}\right)\right]^{\frac{1}{2}} \\
& \alpha_{D o-}=2 \kappa \Lambda^{2} c^{-2}\left[-b-\left(b^{2}-c^{2}\right)\right]^{\frac{1}{2}}
\end{aligned}
$$

$\mathcal{Z}$ it will be zero for the root of $(32)$, so that

$$
\alpha_{Z o}=-2 \kappa b^{-1} \Lambda^{2}
$$

whose location it will be discussed later. From (28) and (29) we have $b>0$, because $\beta>|\gamma|$, and $b>c$. These two last conditions linked with (34) and (35) show that $\alpha_{D o-}<\alpha_{D o+}<0$. So, the $\alpha$-values are located between $\alpha_{D o-}$ and $\alpha_{D o+}$, and becomes $D<0$. Such values belongs to the existence's domain of fixed points $P_{6}, \ldots, P_{9}$, which are real for $\alpha<0$ as we have previously explicited. It is possible to verify that $\alpha_{Z}$ o belongs to the interval $\left(\alpha_{D o-}, \alpha_{D o+}\right)$, that is, between $\left(\bar{\alpha}_{D}\right.$, $\left.\alpha_{D o+}\right)$, where $\bar{\alpha}_{D}=\frac{1}{2}\left(\alpha_{D o+}+\alpha_{D o-}\right)$. Using(34) and (35) we have

$$
\alpha_{Z o}-\alpha_{D o-}=\frac{2 \kappa \Lambda^{2}}{b^{2} c^{2}}\left[b\left(b^{2}-c^{2}\right)+b^{2}\left(b^{2}-c^{2}\right)^{\frac{1}{2}}\right]
$$

The two terms in the right side of (37) are positive because from(28) and (29), $b>0$ and $b>c$. So, $\alpha_{Z o}>\alpha_{D o-}$ and, on the other hand, we see that $\bar{\alpha}_{D}=-\frac{2 \kappa \Lambda^{2}}{c^{2}} b<\alpha_{Z o}$, and thus it shows that $\alpha_{Z o}$ is located to the right of $\bar{\alpha}_{D}$. It is also possible to verify that $\alpha_{Z o}$ is located to the left of $\alpha_{D o+}$. From (34) and (37) we get:

$$
\begin{gathered}
\alpha_{D o+}-\bar{\alpha}_{D}=\frac{2 \kappa \Lambda^{2}}{c^{2}} a_{1} \\
\alpha_{Z_{o}}-\bar{\alpha}_{D}=\frac{2 \kappa \Lambda^{2}}{c^{2}} a_{2}
\end{gathered}
$$

with $a_{1}=\left(b^{2}-c^{2}\right)^{\frac{1}{2}}$ and $a_{2}=\left(b-c^{2} b^{-1}\right)$, being both positive and $a_{1}>a_{2}$, so that $\alpha_{D o+}>\alpha_{Z o}$. So, the eigenvalues can be computed noting the following hypothesis: (I) when $\alpha_{Z_{o}}<\alpha<\alpha_{D o+}$, we have, in this case, $\mathcal{Z}<0$ and $\lambda^{2}=-z \pm i d$, where $z=|\mathcal{Z}|$ and $d=|D|^{\frac{1}{2}}$, such that the four eigenvalues found are conjugate complex, that is, $\lambda_{+1}=\lambda_{-2}^{*}$ and $\lambda_{+2}=\lambda_{-1}^{*}$, with expressions $\lambda_{+1}=r^{\frac{1}{2}}\left(\cos \frac{\theta^{+}}{2}+i \sin \frac{\theta^{+}}{2}\right) ; \lambda_{+2}=$ $-r^{\frac{1}{2}}\left(\cos \frac{\theta^{+}}{2}+i \sin \frac{\theta^{+}}{2}\right) ; \lambda_{-1}=r^{\frac{1}{2}}\left(\cos \frac{\theta^{-}}{2}+i \sin \frac{\theta^{-}}{2}\right)$ and $\lambda_{-2}=-r^{\frac{1}{2}}\left(\cos \frac{\theta^{-}}{2}+i \sin \frac{\theta^{-}}{2}\right)$, where $r=\left(z^{2}+d^{2}\right)^{\frac{1}{2}}$, $\theta^{+}=\tan ^{-1}\left(-\frac{d}{2}\right)+\pi$ e $\theta^{-}=2 \pi-\theta^{+}$, being valid: $\mathcal{R} e\left(\lambda_{+1}\right)=\mathcal{R} e\left(\lambda_{-2}\right)<0$ e $\mathcal{R} e\left(\lambda_{+2}\right)=\mathcal{R} e\left(\lambda_{-1}\right)<0 ;$ (II) when $\alpha_{D o-}<\alpha<\alpha_{Z o}$, the results are equivalent, but in this case $\mathcal{Z}=z$, and not $\mathcal{Z}=-z$, and $\theta^{+}=\tan ^{-1}\left(\frac{d}{2}\right)$ and $\theta^{-}=-\theta^{+}$; (III) when it is considered the hypothesis $D \geq 0$, then $\lambda^{2} \in \mathcal{R}$ and $\lambda$ it will be real or pure imaginary depending on the 
sign of $\lambda^{2}$, which it will be determined by the relation between $\mathcal{Z}^{2}$ and $D$ as showed in (31) and (32), that is, $\mathcal{Z}^{2}-D=\left(b^{2}-c^{2}\right) \alpha^{2} \kappa^{2} \geq 0$, and we can say that the $\operatorname{sign}\left(\lambda^{2}\right)$ is equal to the $\operatorname{sign}(\mathcal{Z})$ and $\lambda^{2} \neq 0, \forall \alpha<0$, so that $\lambda_{+}^{2}=0$ for $\alpha=0$ and $\lambda_{-}^{2}=\mathcal{Z}-D^{\frac{1}{2}}=2 \mathcal{Z}<0$. Then $D \geq 0$ if $\alpha \leq \alpha_{D o-}$ or $\alpha \geq \alpha_{\text {Do+ }}$. When $\alpha \leq \alpha_{\text {Do- }} \Longrightarrow \alpha<\alpha_{Z_{o}} \Longleftrightarrow \mathcal{Z}>0$; if $\alpha \geq \alpha_{D o+} \Longrightarrow \alpha>\alpha_{Z_{o}} \Longleftrightarrow \mathcal{Z}<0$. In summary we see that: (a) $\alpha \leq \alpha_{\text {Do- }} \Longrightarrow \lambda_{+1}=\left(\mathcal{Z}+D^{\frac{1}{2}}\right)^{\frac{1}{2}}>0$; $\lambda_{-1}=\left(\mathcal{Z}-D^{\frac{1}{2}}\right)^{\frac{1}{2}}>0 ; \lambda_{+2}=-\left(\mathcal{Z}+D^{\frac{1}{2}}\right)^{\frac{1}{2}}<0$ and $\lambda_{-2}=-\left(\mathcal{Z}-D^{\frac{1}{2}}\right)^{\frac{1}{2}}<0$, all real; (b) $\alpha_{D o+} \leq$ $\alpha<0 \Longrightarrow \lambda_{+1}=i\left(\left|\mathcal{Z}+D^{\frac{1}{2}}\right|\right)^{\frac{1}{2}} ; \lambda_{-1}=i\left(\left|\mathcal{Z}-D^{\frac{1}{2}}\right|\right)^{\frac{1}{2}}$; $\lambda_{+2}=-i\left(\left|\mathcal{Z}+D^{\frac{1}{2}}\right|\right)^{\frac{1}{2}}$ and $\lambda_{-2}=-i\left(\left|\mathcal{Z}-D^{\frac{1}{2}}\right|\right)^{\frac{1}{2}} ;(\mathbf{c})$ $\alpha=0 \Longrightarrow \lambda_{+1}=\lambda_{+2}=0 ; \lambda_{-1}=-\lambda^{-2}=2 i \Lambda$.

The solution of (24) for the points $P_{2}, \ldots, P_{5}$, in according with the values of its coordinates, are $x_{1}^{*} x_{2}^{*}=0$ and $x_{1}^{* 2}+x_{2}^{* 2}=n^{2}=\frac{-\alpha}{\beta}$, so that substituting these expressions in equations (25)-(27) and, after some algebraic manipulations we find the following characteristic equation:

$\lambda^{4}+2\left(\Lambda^{2}-A^{\prime} \kappa^{-1}\right) \lambda^{2}+\left(\Lambda^{2}+\kappa^{-1} A^{\prime}\right)^{2}-\kappa^{-2} c^{\prime 2} \alpha^{2}=0$

when $A^{\prime}=-b^{\prime} \alpha-\frac{\delta^{2}}{\kappa} ; b^{\prime}=\frac{\gamma+\beta}{2 \beta}=\frac{1}{b} ; c^{\prime}=\frac{3 \beta-\gamma}{2 \beta}$. The equation (40) is equivalent to (24) when substitute $A \rightarrow A^{\prime}, c \rightarrow c^{\prime}$, so that the equations (30)-(32) can be rewritten as :

$$
\begin{gathered}
\lambda^{2}=\mathcal{Z}^{\prime} \pm D^{\prime \frac{1}{2}} \\
D^{\prime}=\frac{c^{\prime 2} \alpha^{2}}{\kappa^{2}}+4 b^{\prime} \alpha \frac{\Lambda^{2}}{\kappa}+4 \Lambda^{4} \\
\mathcal{Z}^{\prime}=\frac{-b^{\prime} \alpha}{\kappa}-2 \Lambda^{2}
\end{gathered}
$$

Although the equations (41)-(43) be similar to (30)(32), the eigenvalues obtained do not present the same features. For the points $P_{6}, \ldots, P_{9}$, the properties of the eigenvalues are strongly influenced by relations $b>0$ and $b>c$ obtained from (28) and (29). In the case of points $P_{2}, \ldots, P_{5}$, despite $b^{\prime}>0$ be valid, meanwhile the relation $b^{\prime}>c^{\prime}$ is not valid. So, from(41)-(43) we have $c^{\prime}>b^{\prime}$ and $b^{\prime}>0$. The first of these two conditions implicates that the equation $D^{\prime}=0$ does not have real roots (the argument in the root of (34) is negative). Being the coefficient of $\alpha^{2}$ in (42) positive, then $D^{\prime}>0$, $\forall \alpha$. It is worth to point out that $\alpha \leq 0$ it is a necessary and sufficient condition for the existence of points $P_{2}, \ldots, P_{5}$ (because $\left.n=\left(\frac{-\alpha}{\beta}\right)^{\frac{1}{2}}\right)$. The minimum of $D^{\prime}$ is found by:

$$
\begin{gathered}
\bar{\alpha}=-2 \kappa b^{\prime} \frac{\Lambda^{2}}{c^{2}}<0 \\
D^{\prime}=4 \Lambda^{4} \frac{c^{\prime 2}-b^{\prime 2}}{c^{\prime 2}}>0
\end{gathered}
$$

and $\mathcal{Z}^{\prime}$ it will be zero for

$$
\alpha_{\mathcal{Z}_{o}^{\prime}}=-2 \kappa b^{\prime} \frac{\Lambda^{2}}{b^{\prime 2}}<0
$$

and from (46), (44) and $c^{\prime}>b^{\prime}:\left|\alpha_{\mathcal{Z}_{o}^{\prime}}\right|>|\bar{\alpha}| \Longrightarrow$ $\alpha_{\mathcal{Z}_{o}^{\prime}}<\bar{\alpha}$ and $\left|\mathcal{Z}^{\prime}\right| \leq\left|D^{\prime \frac{1}{2}}\right|$. Therefore, the eigenvalues are given for $\alpha=0$, as being: $\lambda_{+1}=\lambda_{+2}=0$; $\lambda_{-1}=-\lambda^{-2}=2 i \Lambda$ and for $\alpha<0$, the sign in front of $D^{\prime \frac{1}{2}}$ indicates the sign of $\lambda^{2}$, by virtue of (45), that is: $\lambda_{+1}=\left(\mathcal{Z}^{\prime}+D^{\prime \frac{1}{2}}\right)^{\frac{1}{2}} ; \lambda_{-1}=i\left(\left|\mathcal{Z}^{\prime}-D^{\prime \frac{1}{2}}\right|\right)^{\frac{1}{2}}$; $\lambda_{+2}=-\left(\mathcal{Z}^{\prime}+D^{\prime \frac{1}{2}}\right)^{\frac{1}{2}}$ and $\lambda_{-2}=-i\left(\left|\mathcal{Z}^{\prime}-D^{\prime \frac{1}{2}}\right|\right)^{\frac{1}{2}}$.

In the solution of $(24)$ for the point $P_{1}(0,0,0,0)$ we have: $x_{1}^{*}=x_{2}^{*}=0 ; A_{1}=A_{2}=-\Delta=\alpha-$ $\frac{\delta^{2}}{\kappa}=-b " \alpha-\frac{\delta^{2}}{\kappa}=A ",(b "=-1) ; B=0=c "{ }^{\prime 2} \alpha^{2}$, $\left(c^{\prime \prime}=0\right)$. Substituting, $b \rightarrow-1=b ", c \rightarrow c "=0 \mathrm{e}$ $A \rightarrow A^{\prime \prime}$, in the equations (30)-(32) we get:

$$
\begin{gathered}
\lambda^{2}=\mathcal{Z} " \pm D^{\prime \frac{1}{2}} \\
D^{\prime}=-4 \alpha \frac{\Lambda^{2}}{\kappa}+4 \Lambda^{4} \\
\mathcal{Z} "=\frac{\alpha}{\kappa}-2 \Lambda^{2}
\end{gathered}
$$

and in this case the conditions $(b>0, b>c)$ are not valid. In this case are valid: $b "<0$ and $b "<c "=0$. The eigenvalues will have distinct features of the other two cases already discussed. When $D^{\prime \prime}<0$ : this hypothesis occurs for $\alpha>\alpha_{D_{o}}$ ", where $\alpha_{D_{o}}$ " is the root of equation $D^{\prime \prime}=0$, so that

$$
\begin{gathered}
\alpha_{D_{\circ}}=\frac{\delta^{2}}{\kappa}>0 \\
\mathcal{Z} "=2 \alpha_{D_{\circ}} "
\end{gathered}
$$

and the results are identical to those ones obtained for $D<0$ in the case of points $P_{6}, \ldots, P_{9}$. When $D " \geq 0$ we have $\alpha \leq \alpha_{D_{o}}$ " $=\frac{\delta^{2}}{\kappa}$ and in this case we get the relations $\mathcal{Z} "{ }^{2}-D "=\frac{\alpha^{2}}{\kappa^{2}} \geq 0$ and $|\mathcal{Z} "| \geq D " \frac{1}{2}$. For the computation of eigenvalues we consider, analogously, the cases: $\alpha=0$ and get $\lambda_{+1}=\lambda_{+2}=0 ; \lambda_{-1}=-\lambda^{-2}=2 i \Lambda$; and for $\alpha \neq 0$ we have pure imaginary eigenvalues, that is, $\lambda_{+1}=i\left(\left|\mathcal{Z} "+D^{\prime \prime \frac{1}{2}}\right|\right)^{\frac{1}{2}} ; \lambda_{-1}=i\left(\left|\mathcal{Z} "-D^{\prime \prime \frac{1}{2}}\right|\right)^{\frac{1}{2}}$; $\lambda_{+2}=-i\left(\left|\mathcal{Z} "+D^{\prime \prime} \frac{1}{2}\right|\right)^{\frac{1}{2}}$ and $\lambda_{-2}=-i\left(\left|\mathcal{Z} "-D^{\prime \prime} \frac{1}{2}\right|\right)^{\frac{1}{2}}$. 